Original Research Paper

\title{
A Comprehensive Analysis of Simulated Results for the Performance of AODV and TORA Routing Protocols in Mobile Ad-hoc Networks
}

\author{
${ }^{1}$ Velmurugan Thambusamy and ${ }^{2}$ Navitha Srinivasan \\ ${ }^{1} P G$ and Research Department of Computer Science, D. G. Vaishnav College, Arumbakkam, Chennai-106, India \\ ${ }^{2}$ Department of Computer Science, San Diego Christian College, Manjakudi, India
}

\author{
Article history \\ Received: 25-12-2018 \\ Revised: 25-04-2019 \\ Accepted: 02-05-2019 \\ Corresponding Author: \\ Velmurugan Thambusamy \\ PG and Research Department \\ of Computer Science, \\ D.G.Vaishnav College, \\ Chennai-106, India \\ Email: velmurugan_dgve@yahoo.co.in
}

\begin{abstract}
A Mobile Ad-Hoc Network (MANET) is underpinning less system of movable devices connected by wireless links. Every device in MANET moves arbitrarily on any path with no impediments and this free movement helps in transforming its links to new devices promptly and unpredictably. MANETs do not require a predefined arrangement or centralized administration to interconnect with each other. This research work is carried out to compare the efficiency of two of the on-demand routing protocols - Ad Hoc On-Demand Distance Vector Routing (AODV) and Temporally Ordered Routing Algorithm (TORA) for mobile ad hoc network and the parameters taken into consideration for evaluation are packet delivery ratio, throughput, End to End Delay, Routing overhead, Energy Consumption and others. With the help of NS2, the simulated results of both the protocols are analysed based on its parameters chosen in this work. In particular, the parameters mainly considered for the comparison are packet delivery ratio and End to End Delay. Finally, the performance of these two protocols is compared on the basis of its simulation time.
\end{abstract}

Keywords: Packet Delivery Ratio, Routing Protocols, TORA Protocol, AODV Protocol, Simulation Time

\section{Introduction}

Wireless networks are hasty prominence day by day, as users want wireless connectivity irrespective of their geographic stance. An ad-hoc network is a collection of wireless mobile nodes dynamically forming impermanent network without the use of any existing network infrastructure or centralized administration. Mobile Ad Hoc Networks are self-governing and decentralized wireless systems. MANETs consist of mobile nodes that are free to move in and out in a network. Nodes are the systems or devices i.e. mobile phone, laptop, personal digital assistance, MP3 player and personal computer that are participating in the network and the mobile. These nodes can act as host/router or both at the same time. They can form arbitrary topologies depending on their connectivity with each other in the network. These nodes have the capability to configure themselves and because of their self configuration ability, they can be deployed immediately without the need of any infrastructure.
Internet Engineering Task Force (IETF) has MANET Working Group (WG) that is committed for developing IP routing protocols. Routing protocols is one of the challenging and interesting research areas. Many routing protocols have been developed for MANETS, i.e., AODV, DSR and TORA. In MANETs, due to mobility of nodes, the wireless links are highly error prone and can go down often, obstruction and not as much of infrastructure. Usually, the nodes are mobiles in MANETs. An ad-hoc network is a collection of communication devices called nodes that desire to communicate without any predetermined infrastructure and no pre-defined link organization as shown in Fig. 1.

Two popular MANET routing protocols like $\mathrm{Ad}$ Hoc On-Demand Distance Vector Routing (AODV) (Perkins et al., 2003) and Temporally Ordered Routing Algorithm (TORA) have been implemented in this work. This research work is based on analyzing the ad hoc networks via some of its parameters. In ad-hoc networks, it does not require routers with fixed infrastructure which consist of hosts' interconnection between devices. 


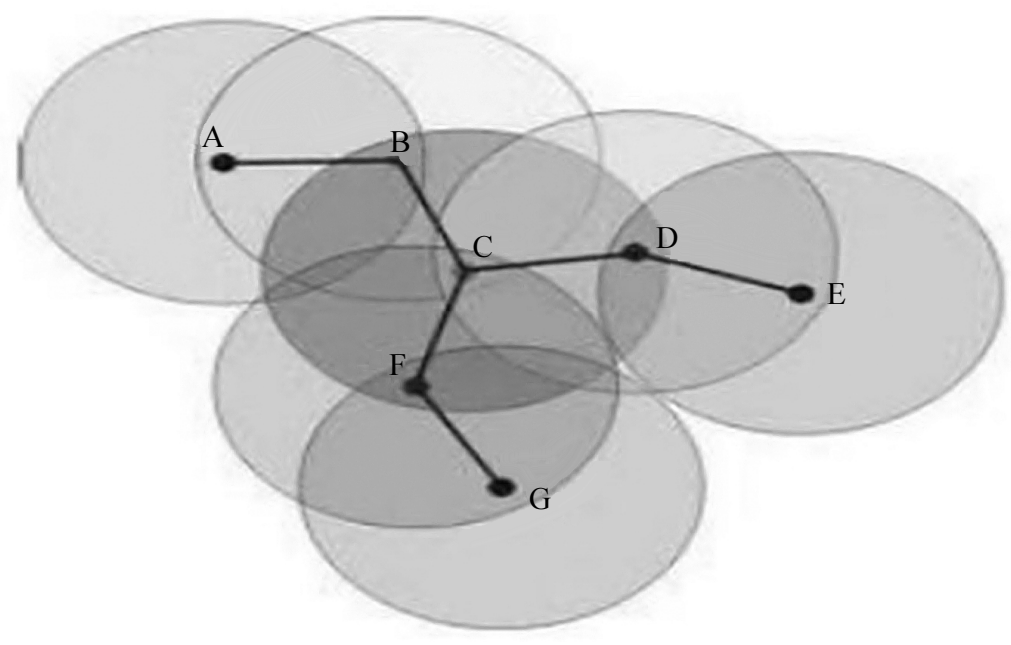

Fig. 1: A mobile ad Hoc network

It can be fixed dynamically. The design features, the routing-based approach, the theoretic information approach, the dynamic control approach, or the game theoretic approach are carried out by the ad-hoc networks. Therefore, the routing protocols need to do four important functions for network topology, maintaining network connection, transmissions planning, channel allocation and packet routing. Routing algorithms have been developed in MANETs based on low control overhead, low processing overhead, multiple hop routing capabilities, dynamic topology maintenance and rotation prevention design goals (Vincent and Corson, 1997).

Sorting out routing protocols in MANETs is based on the routing strategy or network system. According to routing strategy the routing protocols can be classified as table-driven or proactive and source-initiated or reactive or on-demand routing. Each type of protocols differs in different wireless conditions. The performance analysis of these protocols is necessary to work in its behaviour and many factors affect the overall performance of any protocol running on an ad hoc network. For example, the node mobility may cause connection failures, which negatively impact the routing and Quality of Service (QoS) support. Network size, control overhead and traffic intensity can have a significant impact on network measurement. The inherent properties of ad hoc networks may cause unexpected variations in overall network performance. The primary purpose of this paper is to estimate and measure the consequences of many factors affecting network performance and performance analysis of ad-hoc networks. This work emphasizes the performance measurements of delay, through put, route overhead and packet delivery ratio.
The remainder of this paper is organized as follows. Section 2 furnishes survey of different routing protocols used for performance analysis. Section 3 illustrates the simulation methodology and performance metrics. The simulation results and observations are explained in Section 4 followed by the conclusion and future work in Section 5.

\section{Literature Survey}

Many researchers have carried out a number of research works to test the parameters of the routing protocols in MANETs. In such category, some of the previous research articles published in various repositories that are identified and analysed based on the chosen criterion are as follows.

Tariq and Mohammad (2013) have analysed the performance of Standardized Routing Protocols in Adhoc Networks. In this paper, they revise and evaluate the performance of the routing protocols AODV, DSR, DSDV, RAODV, AOMDV and TORA. They have proved that AOMDV has a more improved performance than AODV and RAODV. Lamyaa et al. (2013) have brought out a detailed performance analysis of mobile ad hoc networks under attack. They examined AODV, DSR, TORA and DSDV for MANETs. The authors have zeroed in only on the security concerns under the attack situation in MANET operations. Tuteja et al. (2010) have analyzed a relative performance analysis of AODV, DSDV and DSR routing protocols in MANET using NS2. This paper, compares the mobile ad-hoc network routing protocols DSDV, AODV and DSR. The authors conclude that the performance of all the three protocols is compared with each other to obtain the best performing candidate. Navitha and Velmurugan (2016) proposed the Performance Analysis of AODV and DSR 
routing Protocols in Mobile Ad-hoc Networks. This work measures up to the efficiency of two routing protocols Ad hoc On-Demand Distance Vector (AODV) and Dynamic Source Routing (DSR) based on its performance. Abinaya (2019) discussed more in detail about the application, significant features and different types of protocols in MANET in A Novel Approach to Mobile Ad-hoc Network: Routing Protocols, Characteristics and Features.

The performance analysis has been accomplished on the basis of PDR, Throughput, Delay and Routing overhead as performance parameters. Comparative Analysis of AODV, OLSR, TORA, DSR and DSDV Routing Protocols in Mobile Ad-Hoc Networks discussed by Kaur and Kumar (2013). This research paper revised the characteristics of ad hoc routing protocols Ad-hoc On Demand Distance Vector Routing (AODV), Optimized link State Routing (OLSR), Temporally Ordered Routing Algorithm (TORA), Dynamic Source Routing (DSR), Destination-Sequenced Distance-Vector Routing (DSDV) based on the performance metrics. They proved that TORA performs well in dense networks in terms of packet delivery fraction but at the same time Normalized Routing load of TORA is maximum among all the protocols in both the networks. DSDV has least Normalized Routing load in both low and high traffic. OLSR and DSDV give the least Jitter and Average Delay in both networks.

Performance analysis of AODV, DSR, TORA and OLSR are carried out to achieve group communication in MANET performance by Rajeswari et al. (2012). The main objective of this paper is to achieve robust and reliable group communication in mobile ad hoc network. Performance of the group communication is compared with the given protocols through simulation in NS-2. The analysis is made with respect to the throughput, packet transmission between source and destination. Kumar et al. (2018) proposed Performance Variation of Routing Protocols with Mobility and Scalability in MANET. In this paper they compares the three protocols is done to determine the finest protocol in real-time scenario. Performance when measured on high scalability on a simulation of OLSR protocol as compared to that of AODV and DSR, the results deduced were far better. Patel et al. (2017) have discussed Comparative Performance Analysis of Reactive routing protocols, TORA and AODV: A simulation based evaluation. This paper describes MANET along with the detailed study of protocols named AODV and TORA. NS2 is used to measure the performance of TORA and AODV in the MANET. Research work on three other performance metrics i.e. Network load, jitter, MOS would bring out the contrast between the different mobility models and thus help in making reaching accurate conclusions are carried out by Kaur and Kumar (2014).

\section{The Methodology}

Every routing protocol has its own advantages and disadvantages, none of them can be alleged as utterly better than others. This work selected two reactive routing protocols - AODV and TORA for evaluation based on the simulation results (Samir et al., 2001).

\section{Ad hoc On-demand Distance Vector Routing (AODV)}

Ad-hoc On Demand Distance Vector Routing (AODV) (Perkins and Royer, 1999) is reactive and ondemand routing protocols (Elizabeth and Toh, 1999) where source initiates the route discovery process to find the route to the destination. AODV has three types of control messages for route establishment and maintenance: (1) Route Request (RREQ) (2) RREP (route reply) (3) RERR (Route Error). AODV is competent of both unicast and multicast routing (Liu et al., 2005). It broadcasts a Route Request (RREQ) packet across the network, when a source node desires a route to a destination for which it does not already have a route. Nodes receiving this updated packet information for the source node and backwards pointers have to be set up to the source node in the route tables. In addition to the source node's IP address, current sequence number and broadcast ID, the RREQ also contains the most recent sequence number for the destination of which the source node is alert (Rahman and Zukarnain, 2009).

A node getting the RREQ may send a RREP if it is either the destination or if it has a route to the destination with corresponding sequence number greater than or equal to that contained in the RREQ. If this is the case, it unicasts a RREP back to the source. Otherwise, it rebroadcasts the RREQ. Nodes keep track of the RREQ's source IP address and broadcast ID. If they receive a RREQ which they have already processed, they reject the RREQ and do not forward it. Once the source node receives the RREP, it may begin to forward data packets to the destination as shown in Fig. 2. If a link break occurs while the route is active, the node upstream of the break propagates a RERR message to the source node to inform it of the now unreachable destinations. After receiving the RERR, if the source node still desires the route, it can reinitiate route discovery. Multicast routes are set up in a similar manner.

The sequence numbers are used by AODV to guarantee the freshness of routes. It is loop-free, selfstarting and balance to large numbers of mobile nodes (Kaur and Kumar, 2013; Samir et al., 2001). However, route discovery process is only initiated when routes are not used and they expired and are subsequently discarded. This approach reduces the effects of fusty routes as well as the need for route maintenance for unused routes. Another distinguishing feature of AODV is the ability to provide broadcast, unicast and multicast communication. 


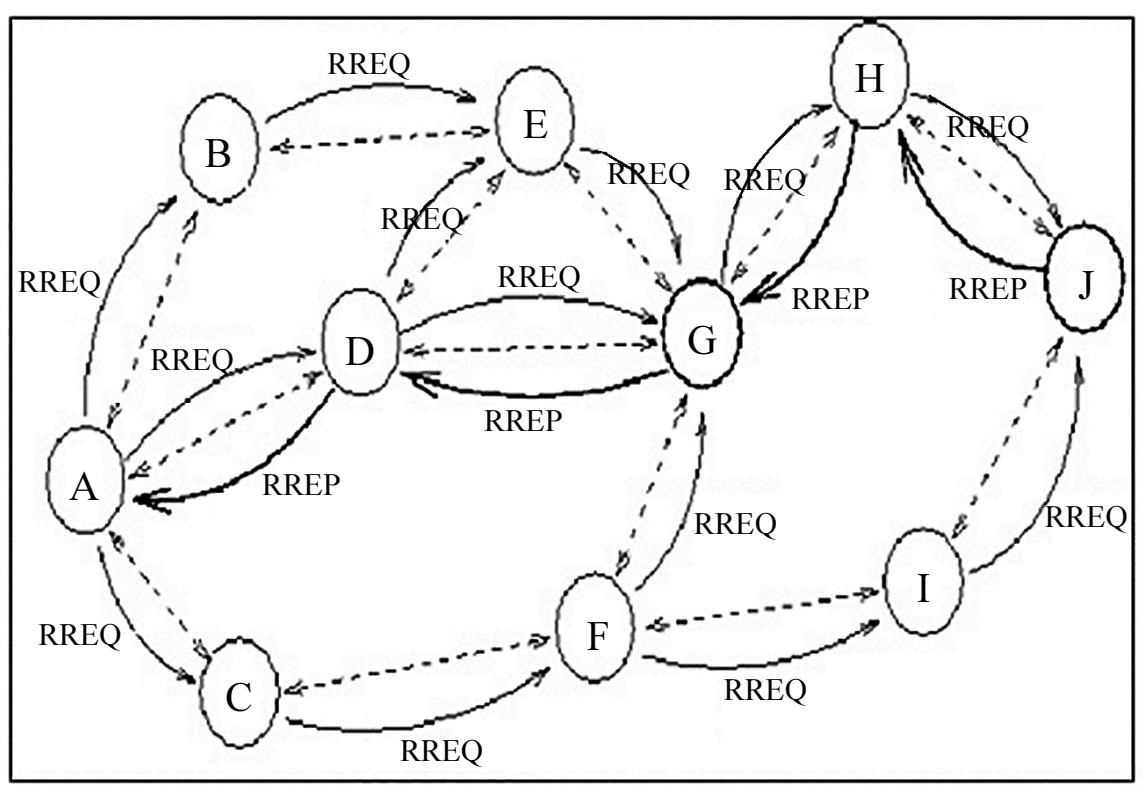

Fig. 2: RREQ and RREP in AODV

AODV uses a broadcast route discovery algorithm and then the unicast route reply massage. AODV (Jiang, 2002) uses different types of messages to discover and maintain links. The benefit of AODV is that it tries to minimize the number of required broadcasts. It creates the routes on an on-demand basis, as opposed to maintain a complete list of routes for each destination. Therefore, the authors of AODV categorize it as a pure on-demand route acquisition system (Perkins and Royer, 1999).

In route discovery, a node broadcasts a Route Request (RREQ) to all nodes in the network till either the destination is reached or another node is found with a valid route entry for the destination whose associated sequence number is at least as great as that contained in RREQ. Then a Route Reply (RREP) is sent back to the source and the discovered route is made available. In route maintenance, when a node detects that a route to a neighbour node is not valid, it removes the routing entry and sends a Route Error (RERR) message to the active neighbours that use the route. This procedure is repeated at nodes that receive RERR messages.

The major difference between AODV and other on demand routing protocols is that it uses a Destination Sequence Number (DestSeqNum) to find out an up-todate path to the destination. A node updates its path destination only if the DestSeqNum of the current packet received is greater than the last DestSeqNum stored at the node. Whenever there is need to create a new route to the destination, the requesting node broadcasts a Route Request (RREQ). A route is determined when this message reaches the next hop node (intermediate node with routing information to the destination) or the destination itself and the Route Reply (RREP) has reached the originator of the request, Routes from the originator of the RREQ to all the nodes that receive this message are cached in these nodes. Whenever there is a link failure, a Route Error (RERR) message is generated. This message contains information about the nodes that are not reachable because of this failure. It also contains IP addresses of all the nodes that using it as their next hop to the destination.

\section{Temporary Ordered Routing Algorithm (TORA)}

The Temporally Ordered Routing Algorithm (TORA) is a source initiated on demand routing protocol. It is a highly adaptive, proficient and scalable distributed routing algorithm based on the link reversal concept and proposed for highly dynamic mobile, multihop wireless networks. TORA searches multiple routes from a source to a destination node. the control messages are localized to a very small set of nodes near the occurrence of a topological change is the most important feature of TORA. To achieve this, the nodes maintain routing information about adjacent nodes. The protocol has three basic functions: Route creation, Route maintenance and Route erasure (Som and Singh, 2012). TORA uses three types of messages to process the functions like QUERY, USER DATAGRAM PROTOCOL and CLEAR:

- The QUERY message for creating a route

- The USER DATAGRAM PROTOCOL message for both creating and maintaining routes

- The CLEAR message for deleting a route

TORA can suffer from unbounded worst-case convergence time for very stressful scenarios (Johnson and Maltz, 1996; Jubin and Tornow, 1987). The key design concept of TORA is the location of control 
messages to a very small set of nodes near the occurrence of a topological change of maintaining multiple routes to the destination so that topological changes do not require any reaction at all. The protocol reacts only when all routes to the destination are lost. In the event of network partitions the protocol is able to detect the partition and erase all invalid routes. Since the shortest path finding is not important in TORA, so the longer paths may lead to loss of information than usual. In TORA longer paths are chosen mainly to reduce the overhead in process of finding new paths (Poornimha, 2017). Table 1 lists some comparisons between the two routing protocols discussed below.

\section{Testing Environment}

In this research paper, Network simulator2 is used for simulating different routing protocols. NS2 is an opensource simulation tool that specifically runs on Linux. The important features of NS2 are, it is a discrete event simulator for networking research and it provides substantial support to simulate group of protocols like Transmission control protocol, file transfer protocol, user datagram protocol, hyper text transfer protocol etc., it also simulates wired and wireless network. Most preferably it uses TCL as its scripting language. In general, NS2 provides users with a way of specifying such network protocols and simulating their corresponding behaviours. Tragic generator and simulated applications are the two types of applications in NS2 particularly famous in the ad hoc networking community. The traffic sources are Continuous Bit Rate (CBR). The source-destination pairs are spread randomly over the network. The mobility model uses 'random waypoint model' in a rectangular filed of $500 \times 500 \mathrm{~m}$ with 75 nodes. In the Random Waypoint model, each node starts to move from its location to a random location with a randomly chosen speed from a minimum speed equal to $5 \mathrm{~m} / \mathrm{s}$ and maximum speed equal to $30 \mathrm{~m} / \mathrm{s}$. In each test, the simulation lasts for $600 \mathrm{sec}$. Once the destination node is reached, the node takes a break for a certain period of time in seconds and another random destination is chosen after that pause time. Different network scenario for different number of nodes and pause times are generated. The model parameters that have been discussed in the following experiments are summarized in Table 2.

\section{Simulation Results and Observations}

The results of AODV and TORA are shown in the Table 1, 2 and 3. The Graphs show comparison between the two protocols by varying different numbers of sources on the basis of the above-mentioned metrics. The experiment of this research work is implemented using NS2. Totally 75 nodes are given to find the performance metrics Packet Delivery Ratio (PDR), Throughput, End to End Delay, Routing overhead and Energy Consumption.

Table 1: Comparison of the two routing protocols

\begin{tabular}{lll}
\hline Parameters & AODV & TORA \\
\hline Source routing & No & No \\
Topology & full & Reduced \\
Update information & Route error & Nodes height \\
Method & Unicast & Broadcast \\
\hline
\end{tabular}

Table 2: Simulation parameter and their values

\begin{tabular}{ll}
\hline Parameter & Values \\
\hline Simulator & NS2 \\
Protocols & AODV and TORA \\
Simulation lost & $200 \mathrm{sec}$ \\
Simulation area & $500 \times 500$ \\
Packet Size & $256,512,625,712,850,1000$ \\
Node placement & Random waypoint \\
Bandwidth & $2 \mathrm{mbps}$ \\
No of mobile nodes & 75 \\
\hline
\end{tabular}

Table 3: AODV-simulation time

\begin{tabular}{lllll}
\hline Time $(\mathrm{sec})$ & PDR & E2E & Rout-over-head & Throughput \\
\hline 5 & 2.1793 & 56.4449 & 3.5921 & 326.582 \\
10 & 2.4485 & 58.7774 & 4.3210 & 321.260 \\
15 & 2.0842 & 56.4651 & 3.2321 & 321.509 \\
20 & 2.0839 & 58.4042 & 3.2201 & 314.646 \\
25 & 2.0981 & 51.7347 & 3.3780 & 54.0525 \\
\hline
\end{tabular}

Table 4; AODV-packet size

\begin{tabular}{llllll}
\hline Size & PDR & E2E & Rout-over-head & Throughput & Egy-consump \\
\hline 256 & 2.0905 & 32.2026 & 3.3330 & 321.9911 & 24.0721 \\
512 & 2.3496 & 51.5004 & 4.2320 & 321.5300 & 35.4236 \\
625 & 1.9894 & 56.7085 & 3.2320 & 322.1670 & 41.5085 \\
712 & 1.9896 & 64.2479 & 3.2316 & 320.9940 & 44.6071 \\
850 & 1.9897 & 72.0306 & 3.2320 & 321.5130 & 51.7443 \\
1000 & 2.0905 & 32.2026 & 3.3330 & 321.9911 & 24.0721 \\
\hline
\end{tabular}


Table 5: AODV-mobility

\begin{tabular}{llllll}
\hline Time $(\mathrm{sec})$ & PDR & E2E & Rout-over-head & Throughput & Egy-consump \\
\hline 5 & 2.0906 & 56.0550 & 3.333 & 321.481 & 41.0876 \\
10 & 2.3496 & 58.9412 & 4.232 & 321.371 & 40.5037 \\
15 & 1.9896 & 56.9526 & 3.232 & 321.956 & 42.4336 \\
20 & 1.9896 & 58.9344 & 3.232 & 321.730 & 41.4493 \\
25 & 1.9583 & 381.046 & 4.061 & 314.280 & 42.7048 \\
\hline
\end{tabular}

Table 6: TORA-simulation time

\begin{tabular}{llllll}
\hline Time $(\mathrm{sec})$ & PDR & E2E & Rout-Over-Head & Throughput & Egy-Consump \\
\hline 5 & 1.7749 & 349.239 & 54.7531 & 1512.06 & 36.5862 \\
10 & 1.6507 & 762.470 & 168.174 & 2683.91 & 90.5917 \\
15 & 1.2703 & 959.512 & 171.204 & 2622.31 & 93.0370 \\
20 & 1.2853 & 772.440 & 154.208 & 2751.04 & 73.1120 \\
25 & 1.3233 & 568.878 & 137.657 & 2955.08 & 46.608 \\
\hline
\end{tabular}

Table 7: TORA-packet size

\begin{tabular}{llllll}
\hline Time $(\mathrm{sec})$ & PDR & E2E & Rout-Over-Head & Throughput & Egy-Consump \\
\hline 5 & 1.7749 & 349.239 & 54.7531 & 1512.06 & 36.5862 \\
10 & 1.6507 & 762.470 & 168.174 & 2683.91 & 90.5917 \\
15 & 1.2703 & 959.512 & 171.204 & 2622.31 & 93.0370 \\
20 & 1.2853 & 772.440 & 154.208 & 2751.04 & 73.1120 \\
25 & 1.3233 & 568.878 & 137.657 & 2955.08 & 46.6080 \\
\hline
\end{tabular}

Table 8: TORA-mobility

\begin{tabular}{llllll}
\hline Time $(\mathrm{sec})$ & PDR & E2E & Rout-over-head & Throughput & Egy-consump \\
\hline 5 & 1.6010 & 292.612 & 137.560 & 5125.78 & 99.7049 \\
10 & 1.7662 & 259.541 & 178.212 & 4481.40 & 98.5565 \\
15 & 1.2604 & 405.597 & 342.638 & 4267.67 & 100.147 \\
20 & 1.1458 & 806.207 & 726.779 & 6075.83 & 99.2404 \\
25 & 1.3333 & 633.524 & 261.702 & 5104.04 & 99.8708 \\
\hline
\end{tabular}

\section{Results of AODV}

Network simulation reproduces the behaviour of a network by means of protocols and here the time taken for simulating the network by considering all these matrices such as Packet delivery ratio, end to end delay, routing overhead, throughput and energy consumption. Hence by considering all these metrics the result of simulation time, packet size and mobility, every $5 \mathrm{sec}$ the packet delivery ratio values increases and throughput will be decreased substantially in AODV. AODV protocol seems to have the best overall performance in simulation experiment. If route discovery in progress, buffering of data packets has a great potential of improvement in AODV. AODV show poor delay characteristics as their routes are typically not the shortest. Even if the initial route discovery phase finds the shortest route, the route may not remain the shortest over a period of time due to node mobility. However, AODV performs a little better delay-wise and can possibly do even better with some fine-tuning of this timeout period by making it a function of node mobility. The results of AODV Vs simulation time, packet size and mobility ratio have been shown in the Tables 3 to 5 .

\section{Results of TORA}

In TORA, the PDR values decrease and end to end delay will be increased drastically. TORA can be quite sensitive to the loss of routing packets compared to the other protocols. TORA has the lengthy delay characteristics because of the loss of distance information with progress. Also in TORA route structure may not take place quickly. This leads to likely lengthy delays while waiting for new routes to be determined. The results of TORA Vs simulation time, packet size and mobility ratio have been shown in the Tables 6 to 8 .

\section{Results and Discussion}

In this research work, the performance of the wireless routing protocols such as AODV and TORA was analyzed using NS-2 simulator. Totally 75 nodes were given to find the performance metrics Packet Delivery Ratio (PDR), Throughput, End to End Delay, Routing overhead and Energy Consumption. This paper reflected the complete simulation results of packet delivery ratio and delay over the routing protocols by varying simulation time. For the most part, AODV protocol is 
better because it has high packet delivery ratio and less delay with low throughput when nodes have high mobility. AODV performs a little better delay and can possible do even better with some fine tuning of this timeout period by making it a function of node mobility. TORA has the least delay characteristics because of the loss of distance information with individual progress have been shown in the following graphs.

The graph shown in Fig. 3 represents performance metrics of AODV Vs Simulation time. Figure 3 illustrates the results of Packet Delivery Ratio with Simulation time. Simulation time and Packet Delivery Ratio are taken along the $\mathrm{x}$-axis and $\mathrm{y}$-axis respectively. These graphs point out that with the low simulation time the packet loss is very high and with the increased simulation time the number of packets received is high. It means that generated packets are being received at a good ratio by the nodes. Also the results of End to End Delay are verified with Simulation time, taking simulation time along the X-axis and End to End Delay in the Y-axis. These graphs designate End to End Delay in ms. In this, the delay is more when the simulation time is low and the delay reduces as the simulation time is high.

Figure 4 exemplify Packet Delivery Ratio which is very poor in TORA. There is always very high packet loss i.e. the number of packets received is low according to simulation time. Significantly, as the simulation time increases the packet loss is high in TORA this graph also indicates End to End Delay in ms. the delay is more when the simulation time is $15 \mathrm{~s}$ and the delay reduces as the simulation time increases.

The Graphs Shown in Fig. 5 typify the performance metrics of AODV Vs Packet Size illustrates the results of Packet Delivery Ratio with Packet Size as well as end to end delay with packet size. This graph depicts PDR as more or less constant for all packet sizes. In this graph, Packet Size and Packet Delivery Ratio are taken along the $\mathrm{x}$-axis and $\mathrm{y}$-axis respectively. Similarly the Packet Size and End to End Delay are also taken along the $\mathrm{x}$ axis and y-axis respectively is shown in Fig. 6. This graph indicates End to End Delay is represented in ms. In this, as per the result, the delay is more as the packet size increases.

The Graphs Shown in Fig. 7 and 8 represents performance metrics of AODV Vs Mobility and TORA Vs Mobility respectively. Figure 7 illustrates the results of Packet Delivery Ratio with Mobility, taking mobility along the $\mathrm{X}$-axis and Packet Delivery Ratio in the $\mathrm{Y}$ axis. In this graph the PDR is constant up to $20 \mathrm{~m} / \mathrm{s}$ and also shows the results of End to End Delay with Mobility, taking Mobility along the $\mathrm{X}$-axis and End to End delay in $\mathrm{Y}$-axis. The unit is measured in ms.

The comparative result analysis of AODV and TORA based on the performance metrics are illustrated in Table 9 and 10 respectively. It also things to see that the various similarities that a protocol exhibits on different simulation parameter taken in consideration. The delay of TORA routing protocol for both default and tuned version is much higher than of AODV. The tuned AODV is better in term of delay. Table 9 and 10 illustrate the results of packets delivery ratio for TORA and AODV protocols respectively. The best PDR results for AODV and TORA are observed by means of delivered packets is reasonably higher in TORA against TORA. Table 9, 10 and Fig. 9, 10 depicts the performance of the protocols under consideration in terms of packets delivery ratio with 75 nodes being the values of number of nodes respectively.

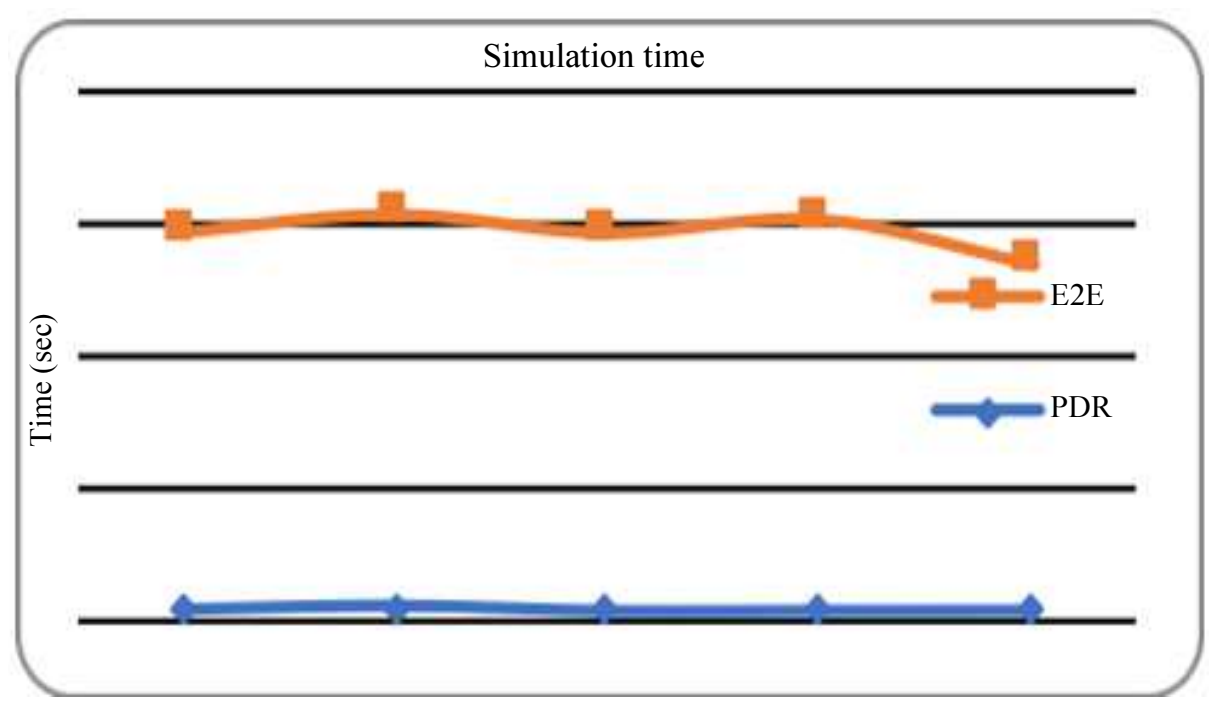

Fig. 3: AODV-simulation time 


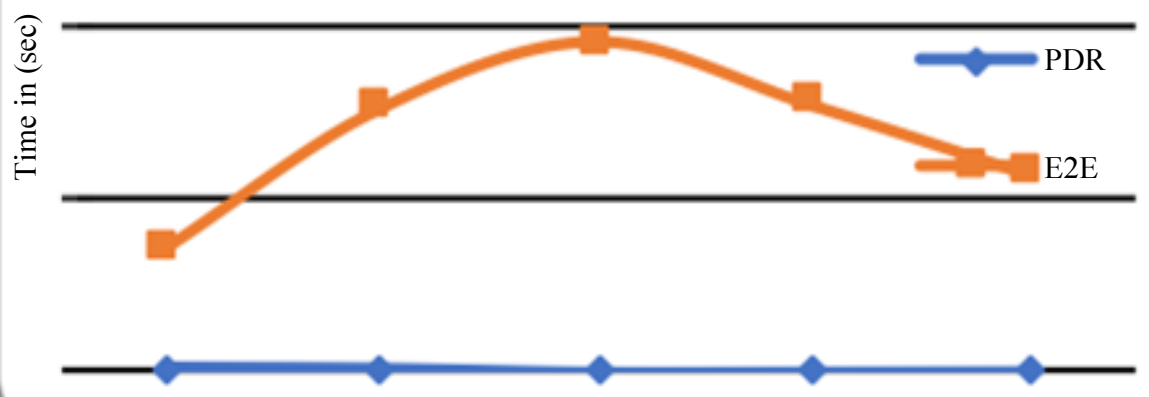

Fig. 4: TORA-simulation time

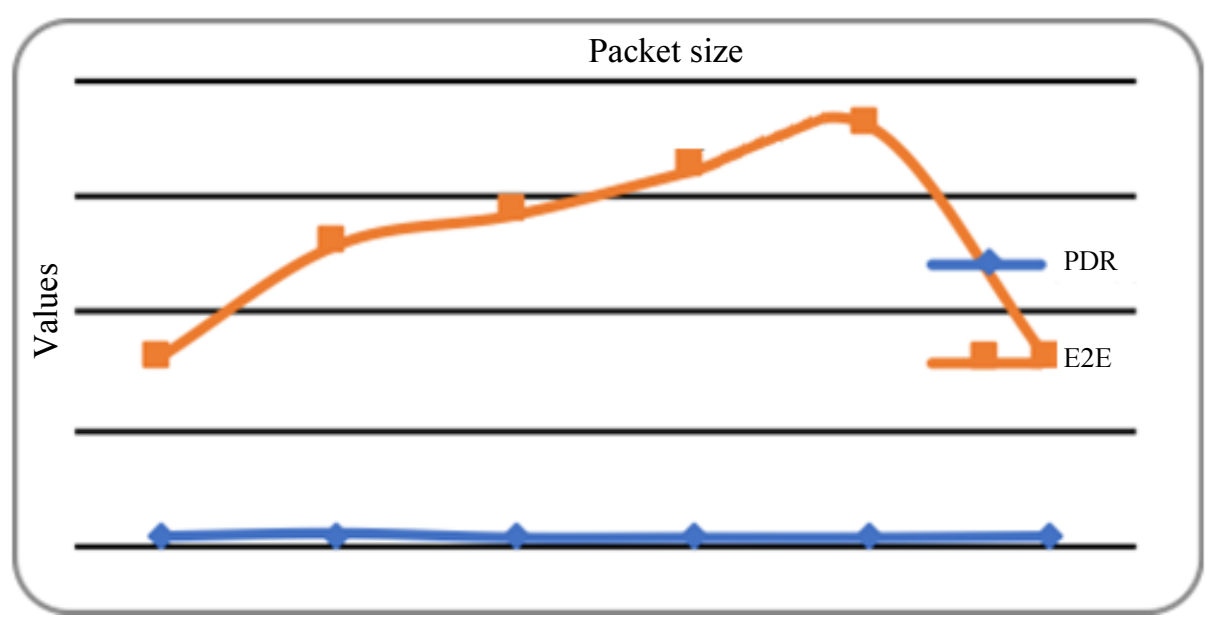

Fig. 5: AODV-packet size

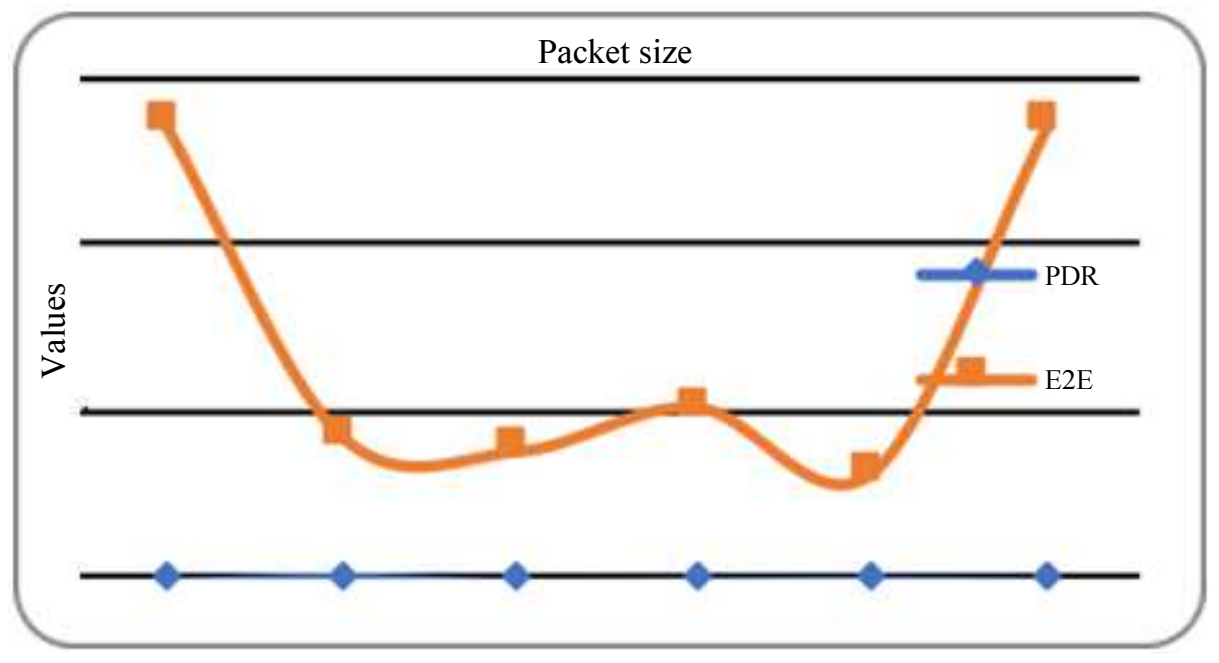

Fig. 6: TORA-packet size 


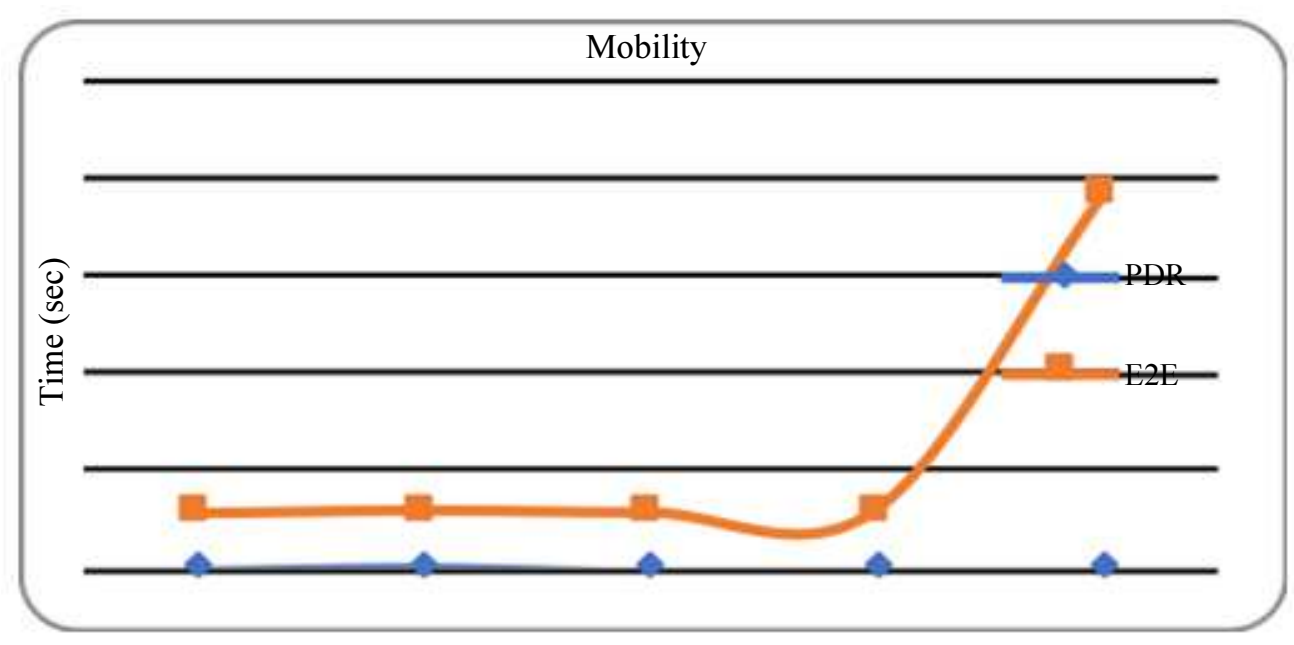

Fig. 7: AODV-mobility

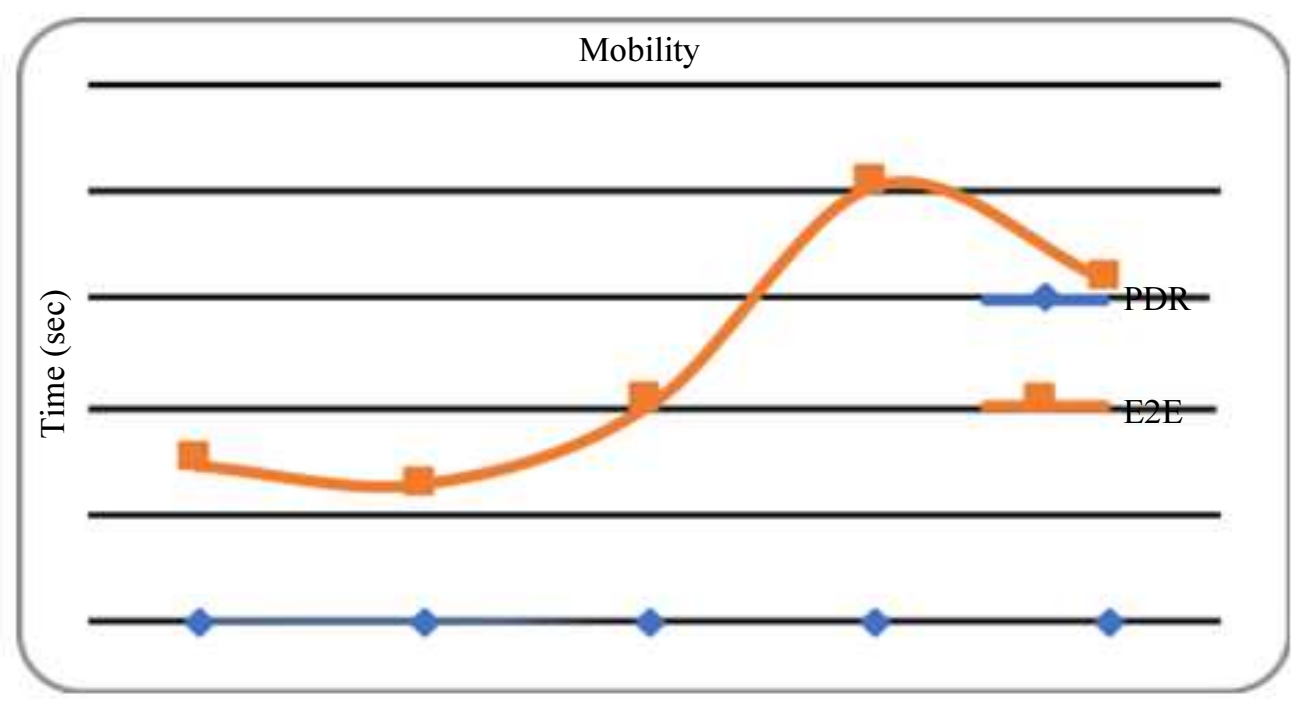

Fig. 8: TORA-mobility

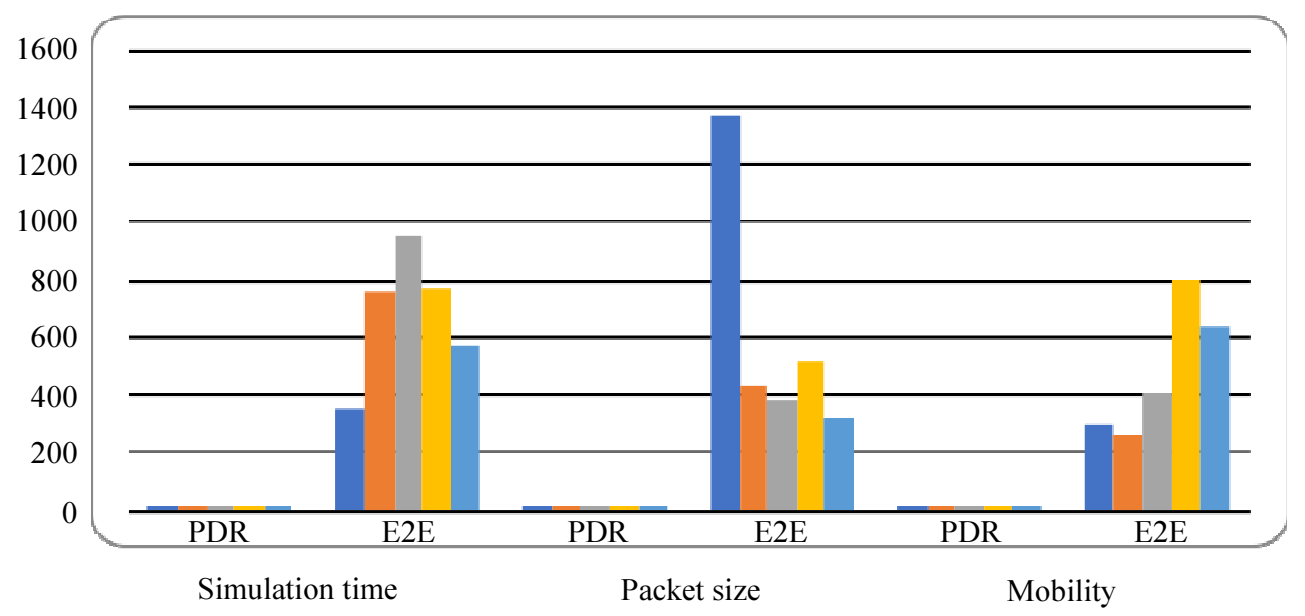

Fig. 9: Consolidated results of TORA 


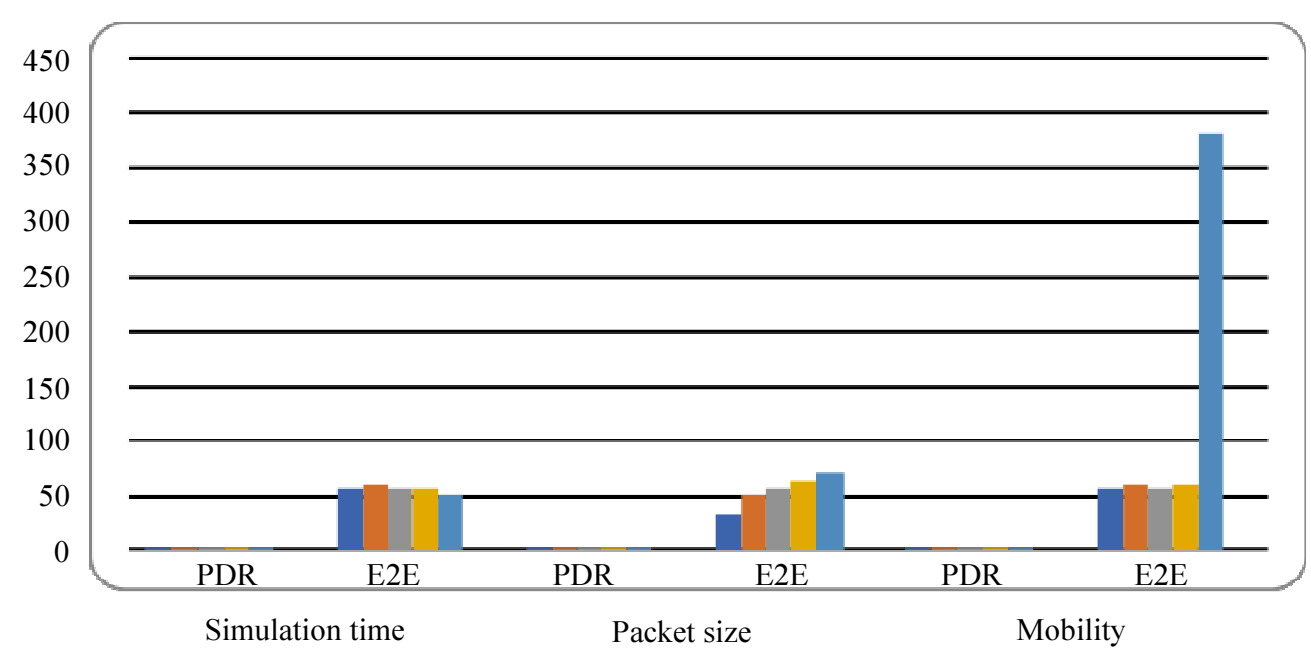

Fig. 10: Consolidated results of AODV

Table 9: Results of TORA protocols

\begin{tabular}{|c|c|c|c|c|c|}
\hline \multicolumn{2}{|c|}{ Simulation time } & \multicolumn{2}{|c|}{ Packet size } & \multicolumn{2}{|c|}{ Mobility } \\
\hline PDR & $\mathrm{E} 2 \mathrm{E}$ & PDR & $\mathrm{E} 2 \mathrm{E}$ & PDR & $\mathrm{E} 2 \mathrm{E}$ \\
\hline 1.7749 & 349.239 & 1.3718 & 1368.42 & 1.601 & 292.612 \\
\hline 1.6507 & 762.470 & 1.7246 & 430.255 & 1.7662 & 259.541 \\
\hline 1.2703 & 959.512 & 1.3125 & 383.678 & 1.2604 & 405.597 \\
\hline 1.2853 & 772.440 & 1.3854 & 515.873 & 1.1458 & 806.207 \\
\hline 1.3233 & 568.878 & 1.4688 & 319.965 & 1.3333 & 633.524 \\
\hline
\end{tabular}

Table 10: Results of AODV protocols

\begin{tabular}{|c|c|c|c|c|c|}
\hline \multicolumn{2}{|c|}{ Simulation time } & \multicolumn{2}{|c|}{ Packet size } & \multicolumn{2}{|c|}{ Mobility } \\
\hline PDR & $\mathrm{E} 2 \mathrm{E}$ & PDR & $\mathrm{E} 2 \mathrm{E}$ & PDR & $\mathrm{E} 2 \mathrm{E}$ \\
\hline 2.1793 & 56.4449 & 2.0905 & 32.2026 & 2.0906 & 56.055 \\
\hline 2.4485 & 58.7774 & 2.3496 & 51.5004 & 2.3496 & 58.9412 \\
\hline 2.0842 & 56.4651 & 1.9894 & 56.7085 & 1.9896 & 56.9526 \\
\hline 2.0839 & 58.4042 & 1.9896 & 64.2479 & 1.9896 & 58.9344 \\
\hline 2.0981 & 51.7347 & 1.9897 & 72.0306 & 1.9583 & 381.046 \\
\hline
\end{tabular}

The simulation time shows that the two metrics of AODV protocol is better than TORA protocol as the nodes are increasing or adding to network. As the nodes are increasing, Packet delivery ratio is high and end-toend delay is low in AODV protocol compared with TORA protocol. Hence efficiency is achieved by the AODV protocol is higher than TORA protocol in mobile ad hoc networks. Furthermore the pictorial chart represents the packet delivery ratio and average end-toend delay for these two protocols and hence comparing them. Here is the graphical representation of the resulting Table 9 and 10.

From the Fig. 9 and 10, it is observed that the packet delivery ratio is very high in case of AODV initially but it decreases substantially if the simulating nodes increase. TORA has a high delay to deliver the packets as compared to AODV. Hence average end to end delay of AODV outperforms the other one. Besides the actual delivery of data packets, the delay time is also affected by route discovery, which is the first step to begin a communication session. By learning the above graphs, the simulation starts all the performance metrics are initially from zero, because initially there is no CBR connection for nodes to take their right place.

\section{Conclusion}

In this research, the performance evaluation has been carried out on AODV and TORA protocols. These routing protocols are evaluated with respect to data packet transmission between source node and destination node using simulation tool NS-2. Also, different metrics are used like end-to-end delay, throughput, packet delivery ratio and energy consumption to investigate the behaviour of these two protocols under different 
simulation time and speeds of the mobile sink. The performance of protocols is evaluated on the basis of various parameters. Totally, 75 numbers of nodes are simulated in the simulation process. From the simulated results, it can be concluded that the mobility model is suitable for small and medium sized networks. The TORA protocol is achieved around 58\% packet delivery ratio when the mobile sink movement speed is $5 \mathrm{~ms}$. But, the AODV got more than that of TORA. Hence, from the results of AODV and TORA, both are compared under normal situations, the AODV is observed as better than the TORA. The AODV protocol is recognized and recommended for the MANETs environment chosen in this simulation. In both scenarios, the AODV is observed as the perfect candidate in terms of its performance via its simulation time. Hence, this work concludes that the On-demand protocol, AODV performed better than the TORA protocol under the taken hypothesis. The future work is to achieve group communication in an efficient way by adding more parameters with these protocols in MANETs.

\section{Acknowledgement}

We thank the reviewers for their careful reading of the paper, their insightful comments and suggestions that greatly improved the manuscript.

\section{Author's Contributions}

Velmurugan Thambusamy: Contributed as the research guide, technical corrections, design the research plan areviewing it critically and contributed to the paper writing.

Navitha Srinivasan: Contribute in writing and formatting of the manuscript and the analysis, organized the study, designed of the proposed work plan development and testing of the application.

\section{Ethics}

This article is original and contains unpublished material. The authors confirm that are no conflict of interest involved and also, the authors confirm that there are no ethical issues involved.

\section{References}

Abinaya, D., 2019. A novel approach to mobile ad-hoc network: Routing protocols, characteristics and features. Int. Res. J. Eng. Technol., 6: 1826-1827.

Elizabeth, M. and R.C.K. Toh, 1999. A review of current routing protocols for ad-hoc mobile wireless networks. IEEE Personal Commun., 6: 46-55.

Jiang, H., 2002. Performance comparison of three routing protocols for ad hoc networks. IEEE J. Comput. Commun. Netw., 3: 547-554.
Johnson, D. and D. Maltz, 1996. Dynamic Source Routing in Ad Hoc Wireless Networks. In: Mobile Computing. The Kluwer International Series in Engineering and Computer Science,, Imielinski $\mathrm{T}$. and H. Korth (Eds.), Springer, Boston, ISBN-10: 978-0-7923-9697-0, pp: 153-181.

Jubin, J. and J.D. Tornow, 1987. The DARPA packet radio network protocols. Proceedings IEEE, 75: 21-32. DOI: 10.1109/PROC.1987.13702

Kaur, A. and R. Kumar, 2014. Comparison study of routing protocol by varying mobility and traffic (CBR, VBR and TCP) using random walk and random way point models. Int. J. Eng. Trends Technol.

Kaur, D. and N. Kumar, 2013. Comparative analysis of AODV, OLSR, TORA, DSR and DSDV routing protocols in mobile ad-hoc networks. Int. J. Comput. Netw. Inform. Security, 3: 39-46.

DOI: $10.5815 /$ ijcnis.2013.03.05

Kumar, M., C. Sharma, A. Dhiman and A.K. Rangra, 2018. Performance Variation of Routing Protocols with Mobility and Scalability in MANET. In: Advances in Intelligent Systems and Computing, Lobiyal, D., V. Mansotra, U. Singh (Eds.), Springer, Singapore, ISBN-10: 978-981-10-6004-5, pp: 9-21.

Lamyaa, M.T.H., M. Tantawy and M. Elsoudani, 2013. Performance of mobile ad hoc networks under attack. Proceedings of the International Conference on Computer Applications Technology, Jan. 20-22, IEEE Xplore Press, Sousse, Tunisia, pp: 1201-1206. DOI: 10.1109/ICCAT.2013.6522034

Liu, Z., M.Z. Kwiatkowska and C. Constantinou, 2005. A biologically inspired QOS routing algorithm for mobile ad hoc networks. Proceedings in 19th International Conference on Advanced Information Networking and Applications, Mar. 28-30, IEEE Xplore Press, Taipei, Taiwan, pp: 426-431. DOI: 10.1109/AINA.2005.13

Navitha, S. and T. Velmurugan, 2016. Performance analysis of AODV and DSR routing protocols in mobile ad-hoc networks. Proceedings of the International Conference on Information and Convergence Technology for Smart Society, (TSS' 16).

Patel, R., B. Patel, S. Patel and A. Parmar, 2017. Comparative performance analysis of reactive routing protocols, TORA and AODV: A simulation based evaluation. Proceedings of the International Conference on Telecommunication, Power Analysis and Computing Techniques, (ACT' 17).

Perkins, C.E. and E.M. Royer, 1999. Ad-hoc on demand distance vector routing. Proceedings of the 2nd IEEE Workshop on Mobile Computing Systems and Applications, Feb. 25-26, IEEE Xplore Press, New Orleans, LA, USA, pp: 90-100.

DOI: 10.1109/MCSA.1999.749281 
Perkins, C.E., E.M. Royer and S. Das, 2003. Ad-hoc OnDemand Distance Vector (AODV). Netw. Work. Group Request Comments.

Poornimha, J., 2017. A survey on performance analysis of TORA with other reactive routing protocols in manets. Int. J. Res. Applied Sci. Eng. Technol., 5: 1817-1818.

Rahman, A.H.A. and Z.A. Zukarnain, 2009. Performance comparison of AODV, DSDV and IDSDV routing protocols in mobile ad hoc networks. Eur. J. Scientific Res., 31: 566-576.

Rajeswari, M., P.U. Maheswari, S. Bhuvaneshwari and S. Gowri, 2012. Performance analysis of AODV, DSR, TORA and OLSR to achieve group communication in MANET. Proceedings of the IEEE- 4th International Conference on Advanced Computing, ICOAC 2012 MIT, Dec. 13-15, IEEE Xplore Press, Chennai, India. DOI: 10.1109/ICoAC.2012.6416834

Samir, R.D., C.E. Perkins and E.E. Royer, 2001. Performance comparison of two on-demand routing protocols for ad hoc networks. IEEE Personal Commun., 8: 16-28.
Som, D.S. and D. Singh, 2012. Performance analysis and simulation of AODV, DSR and TORA routing protocols in MANETs. Int. J. Recent Technol. Eng. 1: 122-127.

Tariq, A.A. and S. Mohammad, 2013. Performance of standardized routing protocols in ad-hoc networks. ICCEEE, 1: 23-28.

Tuteja, A., R. Gujral and S. Thalia, 2010. Comparative performance analysis of DSDV, AODV and DSR routing protocols in MANET using NS2. Proceedings of the International Conference on Advances in Computer Engineering, IEEE Xplore Press, Bangalore, India, pp: 330-333. DOI: 10.1109/ACE.2010.16

Vincent, D.P. and M.S. Corson, 1997. A highly adaptive distributed routing algorithm for mobile wireless networks. Proceedings of the INFOCOM, Apr. 7-11, IEEE Xplore Press, Kobe, Japan. DOI: 10.1109/INFCOM.1997.631180 